\title{
Tecnologias Digitais da Informação e Comunicação na Extensão Universitária: Projeto Redação ENEM*
}

\author{
Juliana Wolf Pereira ${ }^{1}$, Marcelo R. B. Gonçalves ${ }^{1}$, Marilde T. P. Santos ${ }^{2}$, \\ Cláudia R. T. Oliveira ${ }^{1}$, Daniel M. T. Leite ${ }^{1}$, Iara N. S. Lima ${ }^{1}$, Adriana V. Nunes ${ }^{1}$ \\ ${ }^{1}$ Universidade Federal de Mato Grosso do Sul - Campus Coxim (CPCX) \\ Av. Márcio Lima Nantes, S/N - Vila da Barra - \\ Estrada do Pantanal - 79400-000 - Coxim - MS - Brasil \\ ${ }^{2}$ Departamento de Computação \\ Universidade Federal de São Carlos - São Carlos - SP - Brasil \\ \{juliana.wolf, marcelo.barros\}@ufms.br
}

\begin{abstract}
This paper presents an experience report on the development, availability and evaluation of information and communication technologies (ICT) in university extension projects. The present study deals with the design and development of ICT, its applications and analysis of its results in the university extension project - Redação ENEM, between the university and high schools. In evaluation of the ICT in extension actions, the results showed a high degree of satisfaction of the target public reached. This reinforces the positive impact of the use of technology applied to the process of teaching and learning in actions of public policies for society.
\end{abstract}

Resumo. Este trabalho apresenta um relato de experiência do desenvolvimento, disponibilização e avaliação de tecnologias digitais da informação $e$ comunicação (TDIC) em projetos de extensão universitária. O estudo em questão trata da concepção e implementação de TDIC, suas aplicações e análise de seus resultados no Projeto de Extensão Redação ENEM, realizado entre a universidade e escolas de ensino médio. Com a avaliação das TDIC em ações de extensão, os resultados mostraram alto grau de satisfação do público alvo atingido. Isso reforça o impacto positivo do uso da tecnologia aplicada ao processo de ensino e aprendizagem em ações de políticas públicas para a sociedade.

\section{Introdução}

A presença cada vez maior das Tecnologias Digitais da Informação e Comunicação num contexto de ensino e aprendizagem possibilita formas diferenciadas de ensinar e aprender. Os Ambientes Virtuais de Aprendizagem (AVA) e os Recursos Educacionais Abertos (REA) tem sido cada vez mais utilizados com o intuito de beneficiar a relação professor e aluno no processo de ensino e aprendizagem.

No contexto da realização da prova do ENEM, um ambiente virtual acessível com informações, dicas, simulado e correção proporciona um espaço interativo único

\footnotetext{
* Projeto realizado com o apoio da Universidade Federal de Mato Grosso do Sul, PAEXT/2016 (Proc. 215454.1071.2432.30092015)
} 
VI Congresso Brasileiro de Informática na Educação (CBIE 2017)

Anais do XXIII Workshop de Informática na Escola (WIE 2017)

aos alunos e aos professores. Pensando nisso, foi realizado este projeto de extensão entre a universidade e as escolas de ensino médio, utilizando as TDIC, afim de melhor preparar o público alvo para o processo seletivo do ENEM 2016.

Neste trabalho é apresentado um relato de experiência sobre o desenvolvimento, a implantação e a avaliação de TDIC para apoiar o processo de ensino e aprendizagem em ações de extensão do projeto Redação ENEM. Para isso, foram desenvolvidos um AVA para o projeto e três atividades, correção por competência, elaboração de tema de redação e um fórum para contrução colaborativa de temas de redação pelos participantes. Também foi desenvolvido um aplicativo android, que dispõe as informações contidas na cartilha do participante, Redação no ENEM 2016, facilitanto o acesso ao conteúdo. As TDIC foram avaliadas pelos participantes do projeto e os resultados foram positivos.

O artigo está organizado da seguinte maneira: na próxima seção será apresentada a fundamentação teórica deste trabalho; posteriormente, será apresentada a metodologia aplicada. Em seguida, serão apresentadas as seções de concepção e implementação, execução e avaliação do projeto e a análise dos resultados. Por fim, são apresentadas as considerações finais do trabalho.

\section{Fundamentação Teórica}

Nos últimos anos, as tecnologias digitais de informação e comunicação estão sendo cada vez mais utilizadas como apoio ou fim em processos de ensino e aprendizagem. As ferramentas tecnológicas voltadas para o processo ensino e aprendizagem podem dar mais autonomia ao aluno, promovendo um estudo mais focado, intenso e eficiente e, inclusive, subsidiar os processos de avaliação e monitoramento do aprendizado implicando em aprendizagem mais significativa. A utilização e desenvolvimento de ambientes virtuais de aprendizagem (AVA) e de recursos educacionais abertos (REA) tem se tornado fonte de estudo para inúmeros grupos de pesquisa, além de permitir a democratização, universalização e acesso ao conhecimento.

Um AVA é um espaço interativo e dinâmico, que possibilita a disponibilização de diferentes recursos de mídia e permite um processo de ensino e aprendizagem colaborativo, criativo e interessante. De acordo com [Almeida 2010], esses ambientes computacionais colaboram para o suporte de atividades que são mediadas pelas tecnologias de informação e comunicação. Essas tecnologias permitem a integração de variados recursos, linguagens e mídias, além de desenvolver interações entre pessoas e o conhecimento.

O Modular Object Orienteded Distance Learning (Moodle) é uma plataforma Open Source, sendo assim, pode ser instalado, utilizado, modificado e distribuído. Permite o gerenciamento de trabalhos colaborativos, criação e administração de cursos online e comunidades de aprendizagem [Ribeiro et al. 2007]. O Moodle é a plataforma mais utilizada atualmente para o desenvolvimento deste tipo de ambiente por ser um software livre, com uma interface clara, limpa e simples [Galafassi et al. 2013].

Segundo [Lévy 2007], os ambientes colaborativos disponíveis com a web 2.0, possibilitam a disseminação da inteligência coletiva através da interação e a criação colaborativa de conhecimento, permitindo atingir outras pessoas não apenas pelo nome, endereço geográfico ou filiação institucional, mas através dos seus interesses.

O termo recursos educacionais abertos (REA), mais conhecido pela expressão 
VI Congresso Brasileiro de Informática na Educação (CBIE 2017)

Anais do XXIII Workshop de Informática na Escola (WIE 2017)

em inglês Open Educational Resources (OER), apareceu pela primeira vez em uma conferência da UNESCO em 2002. REA são materiais digitais educacionais de forma livre e aberta para uso no ensino, aprendizagem e pesquisa, para toda a comunidade acadêmica [Hylén 2006], [Richter and McPherson 2012].

REA abrange qualquer material educativo, tais como, cursos, módulos de conteúdo, objetos de aprendizagem entre outros. Além das tecnologias e recursos oferecidos livremente e abertamente para apoiar o desenvolvimento, uso, reuso, remixagem, busca, organização e redistribuição de conteúdos [Otsuka et al. 2015]. A utilização e expansão de REA permite a reconstrução de conhecimento colaborativo, e promove a circulação de conhecimento científico formal e o conhecimento popular informal [Okada 2014].

A abordagem de ensino blended learning possibilita o uso de recursos de educação a distância e presencial num processo de ensino e aprendizagem. A experiência educacional promovida pela integração do ensino presencial e de recursos tecnológicos como AVA e REA, permite o enriquecimento e amplia a construção do conhecimento colaborativo [Garrison and Kanuka 2004], [Ramos et al. 2014].

\section{Metodologia}

O Exame Nacional de Ensino Médio (ENEM) surgiu em 1998 e foi criado pelo Ministério da Educação (MEC) do Brasil, com o objetivo de avaliar a qualidade do Ensino Médio no país. A proposta de utilização do ENEM para acesso ao ensino nas instituições federais surgiu no primeiro semestre de 2009. O Ministério da Educação formatou um processo seletivo unificado chamado Sistema de Seleção Unificada (SISU), cujos principais objetivos foram democratizar as oportunidades de acesso às vagas federais de ensino superior, possibilitar a mobilidade acadêmica e induzir a reestruturação dos currículos do ensino médio.

O projeto Redação ENEM é anual e desenvolvido comumente entre os meses de abril e outubro. O projeto é coordenado por professores e realizado parte no ambiente das escolas receptoras, parte nas dependências da própria universidade, especificamente no Laboratório Interdisciplinar de Formação de Educadores (LIFE/CAPES). Os discentes de graduação dos Cursos de Letras e Sistemas de Informação são bolsistas ou colaboradores e participam desde a logística para execução do projeto até o desenvovlvimento e avaliação de AVA e REA, bem como sua execução nas escolas e na correção dos textos.

A metodologia aplicada é constituída de três fases:

1. Concepção e Implementação.

2. Execução e Avaliação do Projeto.

3. Análise dos Resultados.

\section{Concepção e Implementação}

\section{Ambiente Virtual de Aprendizagem - Redação ENEM}

Para o projeto Redação ENEM foi desenvolvido um ambiente virtual de aprendizagem para apoiar o treinamento de corretores. O AVA, Redação ENEM, foi implementado no Moodle e planejado para oferecer suporte aos cursos de treinamento oferecidos pelo 
VI Congresso Brasileiro de Informática na Educação (CBIE 2017)

Anais do XXIII Workshop de Informática na Escola (WIE 2017)

projeto de extensão Redação ENEM a professores das redes pública e privada de ensino. Foram disponibilizados os critérios de correção do ENEM 2016, especificamente na área de Leitura e Produção Textual (redação), por meio de apresentação de mídias e atividades práticas para o aprendizado.

Os treinamentos foram oferecidos tanto no laboratório LIFE/CAPES, quanto nos das escolas atendidas, conforme necessidade. Esperava-se que os docentes participantes tivessem capacidade de corrigir redações conforme os padrões do ENEM e, também, de criar temas para trabalhar tais atividades com seus alunos no decorrer do ano letivo.

Foram propostos três objetos de aprendizagem para ser integrado ao AVA:

- Correção por competência: correção de algumas redações provenientes do simulado, essa atividade é do tipo questionário e cada competência é avaliada numa redação. O corretor atribui uma nota para determinada competência e o resultado é apresentado de imediato com uma justificativa, em caso de acerto ou erro.

- Construindo uma tema de redação: atividade do tipo recurso, em que são apresentados aos professores os critérios e informações para a confecção de um tema de redação no formato do ENEM.

- Fórum sobre a elaboração de temas: atividade do tipo fórum, na qual, os professores podem compartilhar informações para discussão e construção colaborativa de temas de redação.

\section{Aplicativo Redação ENEM}

Para o projeto Redação ENEM foi desenvolvido um aplicativo Android, isto é, um recurso educacional aberto, no qual constam as informações da cartilha do participante Redação no ENEM 2016. Esse aplicativo foi desenvolvido para apoiar um curso de correção de redações do ENEM por docentes da rede pública e privada do ensino médio.

O aplicativo também foi utilizado por estudantes da rede pública durante as ações do projeto Redação ENEM. O aplicativo foi avaliado por docentes e discentes atendidos e os resultados foram motivadores. O aplicativo, conforme figura 1 e figura 2, destaca os principais pontos da Cartilha do Participante Redação ENEM: os critérios para avaliar as competências, as perguntas frequentes e as dicas para a prova.

\section{Execução e Avaliação do Projeto}

De agosto a outubro de 2016 foram realizadas as ações de extensão junto às escolas. Com o AVA e o aplicativo prontos, nesta etapa do projeto foram executadas a palestra motivadora, o curso de correção com os professores e o simulado com os alunos do ensino médio. As ações foram realizadas em duas escolas, uma da rede pública e uma da rede privada.

A Palestra Motivadora ocorreu junto às escolas, com os alunos como público alvo prioritário. Neste momento a Cartilha é discutida pontualmente com os alunos, que podem dispor das informações através do aplicativo. Durante o curso de correção, utilizando o AVA, os professores aprofundam seus conhecimentos sobre as competências. 
VI Congresso Brasileiro de Informática na Educação (CBIE 2017)

Anais do XXIII Workshop de Informática na Escola (WIE 2017)

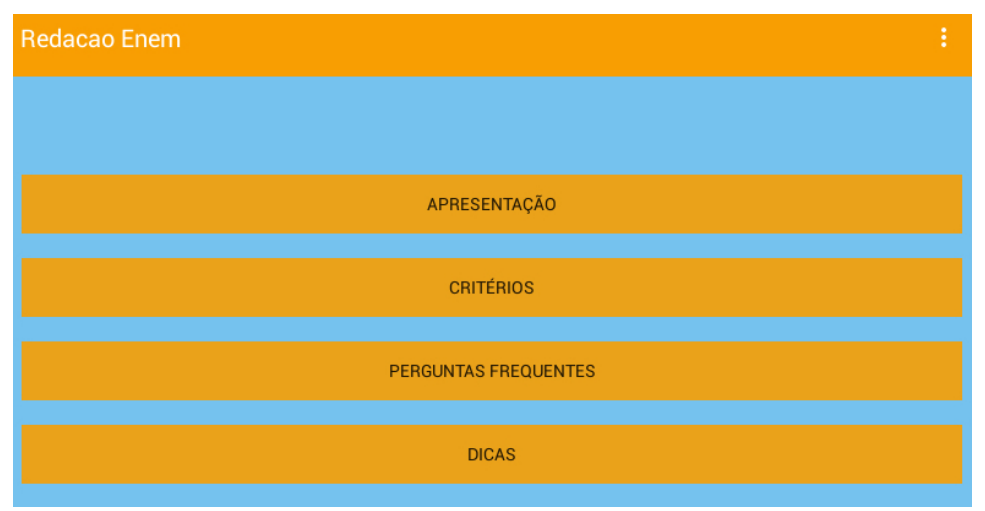

Figure 1. Página Inicial do Aplicativo Redação ENEM

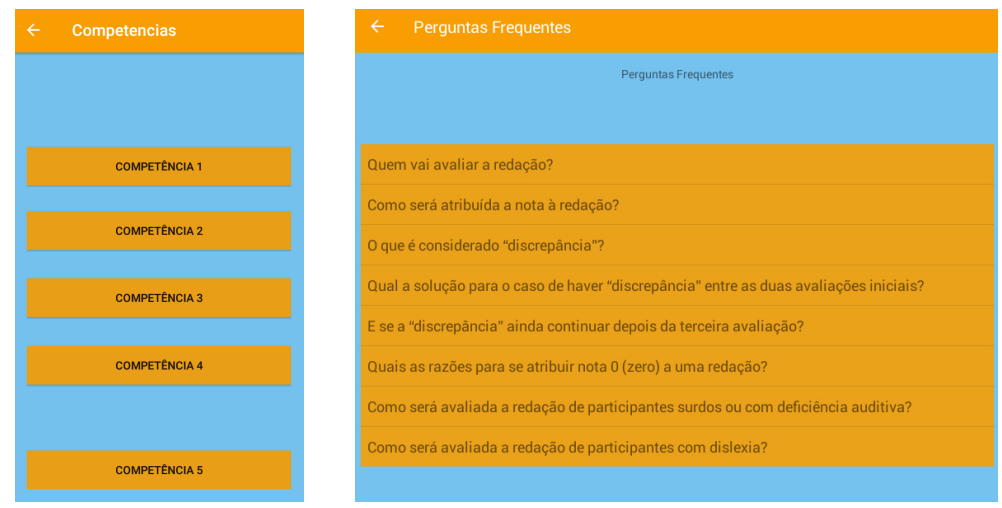

Figure 2. Competências e Perguntas Frequentes

O AVA e o aplicativo Redação ENEM permitiram a prática de uma abordagem blended learning durante o curso de correção por competência, uma vez que os professores podiam utilizar o ambiente e os objetos de aprendizagem disponíveis, tanto in loco quanto depois, on line.

A avaliação foi realizada por meio de questionário aplicado aos participantes do projeto Redação ENEM. Ao todo vinte e seis professores da rede pública e privada participaram do treinamento para correção das redações e na avaliação do projeto. Foram elaboradas questões sobre o conhecimento de informática básica e EAD, sobre o ambiente virtual de aprendizagem, sobre as atividades propostas e sobre o aplicativo.

\section{Análise dos Resultados}

Nesta seção são apresentados os resultados obtidos na análise dos dados gerados com as respostas de um questionário aplicado aos participantes do projeto. $\mathrm{O}$ instrumento utilizado para avaliação foi elaborado pelos autores, considerando os trabalhos descritos em [Franciscato et al. 2008], [Reategui and Finco 2010], [Dillenburg and Teixeira 2011], [Silveira and Carneiro 2012]. A escala de Likert foi utilizada pois permite respostas que variam em gradiência do mais alto grau de satisfação ao mais baixo. Neste trabalho, a variação da escala consiste em:

A - Concordo plenamente

B - Concordo parcialmente 
VI Congresso Brasileiro de Informática na Educação (CBIE 2017)

Anais do XXIII Workshop de Informática na Escola (WIE 2017)
C - Não concordo nem discordo
D - Discordo parcialmente
E - Discordo plenamente

\section{Conhecimento de Informática e Tecnologias}

No grupo analisado, quanto ao acesso à TDIC dos participantes, na Tabela 1, pode-se observar que aproximadamente $45 \%$ dos usuários tinham bom conhecimento de informática. Mais de 50\% conheciam o método de Educação à Distância (EAD), porém, apenas 15,4\% já tiveram acesso ao Moodle antes do projeto. Sobre o uso de redes sociais, mais de $75 \%$ dos usuários acessam com frequência e mais de 50\% usam de forma didática. Em relação aos docentes, apurou-se que aproximadamente $70 \%$ já utilizaram as redes sociais para solucionar dúvidas de alunos. Além disso, as redes sociais mais acessadas pelos participantes foi o WhatsApp para 84,6\%, o Facebook para 76,9\% e o Instagram para 38,5\%.

Table 1. Conhecimento de informática e EAD

\begin{tabular}{|c|c|c|c|c|c|}
\hline Acesso à TDIC & $\mathbf{A}$ & B & $\mathbf{C}$ & $\mathbf{D}$ & $\mathbf{E}$ \\
\hline Conhecimentos em informática & $15,4 \%$ & $30,8 \%$ & $38,5 \%$ & $7,7 \%$ & $7,7 \%$ \\
\hline Conhecimentos de TIC & $7,7 \%$ & $38,5 \%$ & $46,2 \%$ & $7,7 \%$ & $0,0 \%$ \\
\hline Conhecimentos de EAD & $23,1 \%$ & $38,5 \%$ & $30,8 \%$ & $7,7 \%$ & $0,0 \%$ \\
\hline $\begin{array}{l}\text { Utiliza os recursos de EAD na sala de } \\
\text { aula? }\end{array}$ & $7,7 \%$ & $15,4 \%$ & $38,5 \%$ & $30,8 \%$ & $7,7 \%$ \\
\hline Já tinha utilizado o Moodle? & $15,4 \%$ & $7,7 \%$ & $15,4 \%$ & $15,4 \%$ & $46,2 \%$ \\
\hline $\begin{array}{l}\text { Já utilizou alguma rede social para } \\
\text { disponibilizar material didático aos } \\
\text { alunos? }\end{array}$ & $23,1 \%$ & $38,5 \%$ & $15,4 \%$ & $7,7 \%$ & $15,4 \%$ \\
\hline $\begin{array}{l}\text { Já utilizou alguma rede social para solu- } \\
\text { cionar dúvidas do conteúdo estudado com } \\
\text { os alunos? }\end{array}$ & $23,1 \%$ & $46,2 \%$ & $15,4 \%$ & $0,0 \%$ & $15,4 \%$ \\
\hline Frequência de acesso a redes sociais & $46,2 \%$ & $30,8 \%$ & $23,1 \%$ & $0,0 \%$ & $0,0 \%$ \\
\hline $\begin{array}{l}\text { Frequência acesso a redes sociais de } \\
\text { forma didática? }\end{array}$ & $23,1 \%$ & $30,8 \%$ & $38,5 \%$ & $0,0 \%$ & $7,7 \%$ \\
\hline
\end{tabular}

\section{AVA}

O Ambiente Virtual de Aprendizagem (AVA) foi proposto como mediador do ensino, utilizando Tecnologias da Informação e Comunicação (TDIC) para a disseminação do conhecimento aos participantes dos projetos.

Sobre a avaliação do conteúdo apresentado e disponível no AVA, a maioria dos docentes o consideraram relevante, claro, consistente e aplicável. A quantidade de material disponibilizada no AVA foi considerado adequada. Os resultados demonstram que os participantes aprovaram o conteúdo apresentado e disponível no ambiente, conforme Tabela 2. 
VI Congresso Brasileiro de Informática na Educação (CBIE 2017)

Anais do XXIII Workshop de Informática na Escola (WIE 2017)

Table 2. Sobre o conteúdo apresentado e disponível no AVA

\begin{tabular}{||l|c|c|c|c|c||}
\hline \hline \multicolumn{1}{|c|}{$\begin{array}{c}\text { Sobre o conteúdo apresentado e } \\
\text { disponível no ambiente }\end{array}$} & A & B & C & D & E \\
\hline \hline Relevância & $46,2 \%$ & $30,8 \%$ & $15,4 \%$ & $7,7 \%$ & $0,0 \%$ \\
\hline Clareza e consistência & $53,8 \%$ & $23,1 \%$ & $7,7 \%$ & $15,4 \%$ & $0,0 \%$ \\
\hline Aplicabilidade & $38,5 \%$ & $38,5 \%$ & $7,7 \%$ & $15,4 \%$ & $0,0 \%$ \\
\hline Material & $46,2 \%$ & $23,1 \%$ & $23,1 \%$ & $7,7 \%$ & $0,0 \%$ \\
\hline Conceito & $53,8 \%$ & $23,1 \%$ & $15,4 \%$ & $7,7 \%$ & $0,0 \%$ \\
\hline Contextualização & $53,8 \%$ & $23,1 \%$ & $15,4 \%$ & $7,7 \%$ & $0,0 \%$ \\
\hline Qualidade & $53,8 \%$ & $30,8 \%$ & $7,7 \%$ & $7,7 \%$ & $0,0 \%$ \\
\hline \hline
\end{tabular}

A Tabela 3 apresenta as respostas que se referem à interação dos participantes com o ambiente. A avaliação foi muito positiva em relação aos seguintes aspectos indagados: facilidade, interatividade e relevância, avaliados como "concordo plenamente" por $75 \%$ dos participantes. Inclui-se, ainda, a avaliação sobre as cores, letras e tópicos, os quais foram considerados "concordo plenamente" por $62,5 \%$ dos participantes, verificando-se o mesmo quanto ao quesito "replicável".

Table 3. Sobre interação com o AVA

\begin{tabular}{||l|c|c|c|c|c||}
\hline \multicolumn{1}{|c|}{ Sobre interação com o ambiente } & A & B & C & D & E \\
\hline \hline Facilidade & $61,5 \%$ & $15,4 \%$ & $15,4 \%$ & $7,7 \%$ & $0,0 \%$ \\
\hline Interatividade & $53,8 \%$ & $30,8 \%$ & $7,7 \%$ & $7,7 \%$ & $0,0 \%$ \\
\hline Visualmente atraente & $38,5 \%$ & $38,5 \%$ & $15,4 \%$ & $7,7 \%$ & $0,0 \%$ \\
\hline Espaço na tela & $30,8 \%$ & $53,8 \%$ & $7,7 \%$ & $7,7 \%$ & $0,0 \%$ \\
\hline Cores, Letras e Tópicos & $46,2 \%$ & $38,5 \%$ & $7,7 \%$ & $7,7 \%$ & $0,0 \%$ \\
\hline
\end{tabular}

Pode-se considerar que os demais quesitos também foram avaliados positivamente, o item visualmente atraente teve concordância de quase $80 \%$. A avaliação feita demonstra alto nível de satisfação quanto à interação e facilidade no uso do ambiente. A maioria concordou, ainda, com a distribuição no espaço da tela e com as cores, letras e tópicos.

\section{Atividades propostas}

Na tabela 4 são demonstrados os resultados da avaliação sobre a atividade de correção por competência de redações provenientes do simulado, a mesma foi considerada relevante, replicável, o seu método tido como interessante, bem como foi considerada como instrumento que reforça conceitos e ajuda a desenvolver habilidades por aproximadamente $70 \%$ dos participantes. O quesito sobre a relevância da atividade obteve a melhor avaliação, assim considerado por mais de $80 \%$ dos participantes. O único aspecto julgado pela maioria como apenas "concordo parcialmente"condiz ao tempo de resposta, desta maneira julgado por $53,8 \%$ dos avaliadores. 
VI Congresso Brasileiro de Informática na Educação (CBIE 2017)

Anais do XXIII Workshop de Informática na Escola (WIE 2017)

Table 4. Avaliação da atividade correção por competência

\begin{tabular}{||l|c|c|c|c|c||}
\hline \hline $\begin{array}{c}\text { Objeto de aprendizagem: correção por } \\
\text { competência }\end{array}$ & $\mathbf{A}$ & $\mathbf{B}$ & $\mathbf{C}$ & $\mathbf{D}$ & $\mathbf{E}$ \\
\hline \hline Relevante & $61,5 \%$ & $23,1 \%$ & $7,7 \%$ & $7,7 \%$ & $0,0 \%$ \\
\hline Replicável & $53,8 \%$ & $23,1 \%$ & $15,4 \%$ & $7,7 \%$ & $0,0 \%$ \\
\hline O método da atividade foi interessante & $61,5 \%$ & $23,1 \%$ & $7,7 \%$ & $7,7 \%$ & $0,0 \%$ \\
\hline Reforça conceitos & $61,5 \%$ & $23,1 \%$ & $7,7 \%$ & $7,7 \%$ & $0,0 \%$ \\
\hline Tempo de resposta & $15,4 \%$ & $53,8 \%$ & $15,4 \%$ & $15,4 \%$ & $0,0 \%$ \\
\hline $\begin{array}{l}\text { Ajudou a desenvolver habilidades sobre o } \\
\text { assunto }\end{array}$ & $53,8 \%$ & $23,1 \%$ & $23,1 \%$ & $0,0 \%$ & $0,0 \%$ \\
\hline \hline
\end{tabular}

Na tabela 5 é demonstrado o resultado da avaliação sobre a atividade de correção por competência de redações do simulado, a mesma foi considerada relevante, replicável, o seu método tido como interessante, bem como foi considerada como instrumento que reforça conceitos e ajuda a desenvolver habilidades por mais de $70 \%$ dos participantes.

Table 5. Avaliação da atividade elaboração de um tema

\begin{tabular}{||l|c|c|c|c|c||}
\hline \hline $\begin{array}{c}\text { Objeto de aprendizagem: elaboração } \\
\text { de um tema }\end{array}$ & A & B & C & D & E \\
\hline \hline Relevante & $38,5 \%$ & $30,8 \%$ & $23,1 \%$ & $7,7 \%$ & $0,0 \%$ \\
\hline Replicável & $46,2 \%$ & $30,8 \%$ & $15,4 \%$ & $7,7 \%$ & $0,0 \%$ \\
\hline O método da atividade foi interessante & $53,8 \%$ & $38,5 \%$ & $7,7 \%$ & $0,0 \%$ & $0,0 \%$ \\
\hline Reforça conceitos & $53,8 \%$ & $38,5 \%$ & $7,7 \%$ & $0,0 \%$ & $0,0 \%$ \\
\hline Tempo de resposta & $23,1 \%$ & $38,5 \%$ & $38,5 \%$ & $0,0 \%$ & $0,0 \%$ \\
\hline $\begin{array}{l}\text { Ajudou a desenvolver habilidades sobre o } \\
\text { assunto }\end{array}$ & $53,8 \%$ & $38,5 \%$ & $7,7 \%$ & $0,0 \%$ & $0,0 \%$ \\
\hline \hline
\end{tabular}

\section{Aplicativo}

O aplicativo Redação ENEM foi proposto como mediador do ensino, utilizando TDIC para a disseminação do conhecimento aos participantes dos projetos. Na tabela 6 são apresentados os resultados das respostas dos participantes dos questionamentos sobre o aplicativo.

Como pode ser observado na tabela 6, o aplicativo Redação ENEM é fácil de utilizar e funciona corretamente para a maioria dos participantes. Para quase $70 \%$ as informações apresentadas são relevantes e auxiliam na realização de atividades de construção e de correção de redações.

\section{Considerações Finais}

Este trabalho apresentou um relato de experiência da utilização de TDIC para o Projeto de Extensão Redação ENEM 2016. Para o projeto foram desenvolvidas atividades num 
VI Congresso Brasileiro de Informática na Educação (CBIE 2017)

Anais do XXIII Workshop de Informática na Escola (WIE 2017)

Table 6. Avaliação do aplicativo

\begin{tabular}{||l|c|c|c|c|c||}
\hline \multicolumn{1}{|c|}{ Aplicativo Redação ENEM } & A & B & C & D & E \\
\hline \hline Funciona corretamente & $46,2 \%$ & $30,8 \%$ & $15,4 \%$ & $7,7 \%$ & $0,0 \%$ \\
\hline Fácil de utilizar & $53,8 \%$ & $23,1 \%$ & $23,1 \%$ & $0,0 \%$ & $0,0 \%$ \\
\hline $\begin{array}{l}\text { Permite mais interatividade ao conteúdo } \\
\text { impresso }\end{array}$ & $61,5 \%$ & $0,0 \%$ & $30,8 \%$ & $7,7 \%$ & $0,0 \%$ \\
\hline $\begin{array}{l}\text { As informações são suficientes para o en- } \\
\text { tender o funcionamento }\end{array}$ & $46,2 \%$ & $23,1 \%$ & $30,8 \%$ & $0,0 \%$ & $0,0 \%$ \\
\hline $\begin{array}{l}\text { Fornece feedback apropriado sobre a ação } \\
\text { do usuário }\end{array}$ & $38,5 \%$ & $30,8 \%$ & $30,8 \%$ & $0,0 \%$ & $0,0 \%$ \\
\hline $\begin{array}{l}\text { As informações apresentadas são rele- } \\
\text { vantes }\end{array}$ & $53,8 \%$ & $15,4 \%$ & $30,8 \%$ & $0,0 \%$ & $0,0 \%$ \\
\hline $\begin{array}{l}\text { Utilizar o aplicativo off-line auxilia na } \\
\text { realização de atividades }\end{array}$ & $46,2 \%$ & $23,1 \%$ & $30,8 \%$ & $0,0 \%$ & $0,0 \%$ \\
\hline $\begin{array}{l}\text { Mobilidade para consultas às instruções } \\
\text { do guia de redação }\end{array}$ & $30,8 \%$ & $38,5 \%$ & $30,8 \%$ & $0,0 \%$ & $0,0 \%$ \\
\hline $\begin{array}{l}\text { A disposição de menus e recursos fa- } \\
\text { vorece a utilização do aplicativo }\end{array}$ & $41,7 \%$ & $25 \%$ & $33,3 \%$ & $0,0 \%$ & $0,0 \%$ \\
\hline $\begin{array}{l}\text { A utilização do aplicativo facilita a } \\
\text { correção de redações }\end{array}$ & $50 \%$ & $16,7 \%$ & $33,3 \%$ & $0,0 \%$ & $0,0 \%$ \\
\hline $\begin{array}{l}\text { É necessário aprender muitos conceitos } \\
\text { para saber utilizar o aplicativo }\end{array}$ & $46,2 \%$ & $7,7 \%$ & $30,8 \%$ & $7,7 \%$ & $7,7 \%$ \\
\hline $\begin{array}{l}\text { Facilmente por qualquer usuário, pois não } \\
\text { exige conhecimentos técnicos. }\end{array}$ & $38,5 \%$ & $23,1 \%$ & $38,5 \%$ & $0,0 \%$ & $0,0 \%$ \\
\hline \hline
\end{tabular}

ambiente virtual de aprendizagem e um aplicativo android. Para o AVA foram propostos três objetos de aprendizagem distintos que, em conjunto com o aplicativo, tornaram a correção por competência e a construção de temas para redação mais dinâmicas e colaborativas. Ambos os recursos permitiram uma maior interação entre os participantes do projeto, acusando uma melhoria na relação de ensino e aprendizagem.

A avaliação do trabalho foi realizada através da análise dos questionários preenchidos pelos participantes do projeto. As questões tratavam sobre o conhecimento em informática e em EAD, sobre conteúdo disponibilizado no AVA e sobre a interação com o AVA. Os conteúdos foram considerados relevantes, contextualizados e de qualidade. Foi ainda indicada a facilidade e interatividade com o AVA. Um dos itens a ser aprimorado é a interface com o usuário que deve ser visualmente mais atraente.

As atividades propostas no ambiente virtual foram consideradas relevantes e replicáveis, bem como o método foi considerado interessante. No contexto de redação ENEM, a avaliação demostrou que as atividades ajudaram a desenvolver habilidades sobre o assunto. $\mathrm{O}$ aplicativo foi considerado de fácil utilização, com informações relevantes 
VI Congresso Brasileiro de Informática na Educação (CBIE 2017)

Anais do XXIII Workshop de Informática na Escola (WIE 2017)

e facilitador do processo de correção de redações.

Para os trabalhos futuros pretende-se aprimorar o AVA, desenvolver novos REA e executar a proposta em outras escolas.

\section{References}

Almeida, M. E. B. d. (2010). Tecnologia e educação a distância: Abordagens e contribuições dos ambientes digitais e interativos de aprendizagem. Revista Brasileira de Educação a Distância, page 6.

Dillenburg, D. J. and Teixeira, A. C. (2011). Uma proposta de avaliação qualitativa em ambientes virtuais de aprendizagem. In Brazilian Symposium on Computers in Education (Simpósio Brasileiro de Informática na Educação-SBIE), volume 1.

Franciscato, F. T., Ribeiro, P. d. S., Mozzaquatro, P. M., and Medina, R. D. (2008). Avaliação dos ambientes virtuais de aprendizagem moodle, teleduc e tidia-ae: um estudo comparativo, ppgi/ufsm. RENOTE, 6(2).

Galafassi, F. P., Gluz, J. C., and Galafassi, C. (2013). Análise crítica das pesquisas recentes sobre as tecnologias de objetos de aprendizagem e ambientes virtuais de aprendizagem. Revista Brasileira de Informática na Educação, 21(3):41-52.

Garrison, D. R. and Kanuka, H. (2004). Blended learning: Uncovering its transformative potential in higher education. The internet and higher education, 7(2):95-105.

Hylén, J. (2006). Open educational resources: Opportunities and challenges. Proceedings of Open Education, pages 49-63.

Lévy, P. (2007). A Inteligência coletiva. Edições Loyola.

Okada, A. (2014). Mapas do conhecimento com recursos educacionais abertos aplicados à coaprendizagem baseada em coinvestigação.

Otsuka, J. L., dos Santos, E. G. C., de Souza Gracioso, L., Rosales, G. C. M., and Kawakami, C. (2015). Livre saber (lisa): um repositório de recursos educacionais abertos de cursos a distância. Revista Brasileira de Informática na Educação, 23(1).

Ramos, J. L. C., da Silva, R. F. P., Silva, J. C. S., and Gomes, A. S. (2014). Adoção de blended learning: verificação do potencial de ampliação na universidade federal do vale do são francisco. In Anais do Workshop de Informática na Escola, volume 20, page 36 .

Reategui, E. and Finco, M. D. (2010). Proposta de diretrizes para avaliação de objetos de aprendizagem considerando aspectos pedagógicos e técnicos. RENOTE, 8(3).

Ribeiro, E. N., Mendonça, G. d. A., and Mendonça, A. F. (2007). A importância dos ambientes virtuais de aprendizagem na busca de novos domínios da ead. In Anais do $13^{\circ}$ Congresso Internacional de Educação a Distância. Curitiba, Brasil.

Richter, T. and McPherson, M. (2012). Open educational resources: education for the world? Distance education, 33(2):201-219.

Silveira, M. S. and Carneiro, M. L. F. (2012). Diretrizes para a avaliação da usabilidade de objetos de aprendizagem. In Brazilian Symposium on Computers in Education (Simpósio Brasileiro de Informática na Educação-SBIE), volume 23. 